\title{
Entrelacs
}

Cinéma et audiovisuel

\section{De la prise de son à la diffusion: mystères et mécanique de la perspective sonore.}

Sound recording and sound diffusion : enigmas and engineering of sound perspective.

\section{A. Beznosiuk}

\section{OpenEdition}

\section{Journals}

Édition électronique

URL : http://journals.openedition.org/entrelacs/2115

DOI : 10.4000/entrelacs.2115

ISSN : 2261-5482

Éditeur

Éditions Téraèdre

\section{Référence électronique}

A. Beznosiuk, " De la prise de son à la diffusion: mystères et mécanique de la perspective sonore. », Entrelacs [En ligne], 13 | 2017, mis en ligne le 18 mai 2017, consulté le 19 avril 2019. URL : http:// journals.openedition.org/entrelacs/2115; DOI : 10.4000/entrelacs.2115

Ce document a été généré automatiquement le 19 avril 2019

Tous droits réservés 


\title{
De la prise de son à la diffusion: mystères et mécanique de la perspective sonore.
}

\author{
Sound recording and sound diffusion : enigmas and engineering of sound \\ perspective.
}

\section{A. Beznosiuk}

1 Lors des différentes étapes de la production sonore, de la prise de son à la diffusion en passant par le montage, le traitement en post-production et le mixage, la question de la représentation de l'espace se pose de façon toujours singulière et nécessite, outre la mise en œuvre de systèmes complexes, d'intégrer les mystérieuses mécaniques qui président chez l'auditeur ou l'audio-spectateur à la construction de la représentation de l'espace sonore.

De surcroit, nous ne disposons pas de la possibilité d'arrêter notre attention sur un événement ponctuel qui serait particulièrement significatif. Les évènements sonores se déroulant selon un flux dont nous sommes prisonniers;

en effet, les stimuli auditifs nous parviennent pour certains séquentiellement et pour d'autres simultanément. Nous déployons donc une capacité à isoler telle ou telle grandeur, plus précisément ses variations, et dans le même temps, nous procédons à une réception englobant la somme de ces informations.

2 La fenêtre temporelle d'intégration des informations sonores est manifestement variable et la construction de la représentation de l'espace mobilise très largement cette capacité.

3 Rappelons ici les grandeurs fondamentales de la perception auditive qui vont participer à l'élaboration de l'image spatiale :

\section{La sensation de niveau:}

4 Nous sommes inégaux en terme de sensibilité aux faibles niveaux sonores mais même atteints de presbyacousie, l'être humain présente un haut niveau de performance de 
perception différentielle; un écart de niveau, même faible entre deux sons sera perçu de façon sensible.

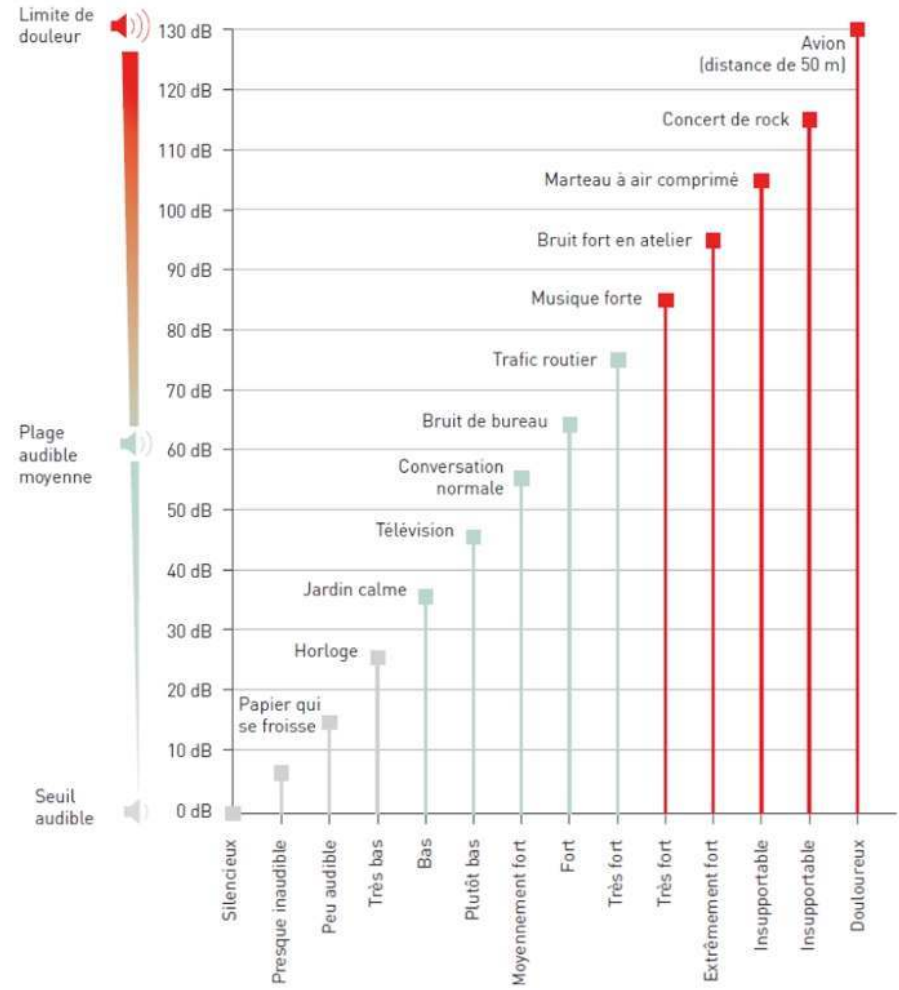

Fig 1 : échelle de perception du niveau sonore

http://auriol.free.fr/psychosonique/ClefDesSons/seuil.htm

5 Notons ici la performance du système auditif qui présente une échelle de perception variant sur un rapport de 1 pour $1 \times 10^{6}$. La sensation de décroissance du niveau d'une source, ou sa comparaison avec une source plus puissante participe très largement à la construction de la représentation spatiale, tant en terme de position de l'auditeur dans l'espace que de localisation des sources. 


\section{La sensation de hauteur.}

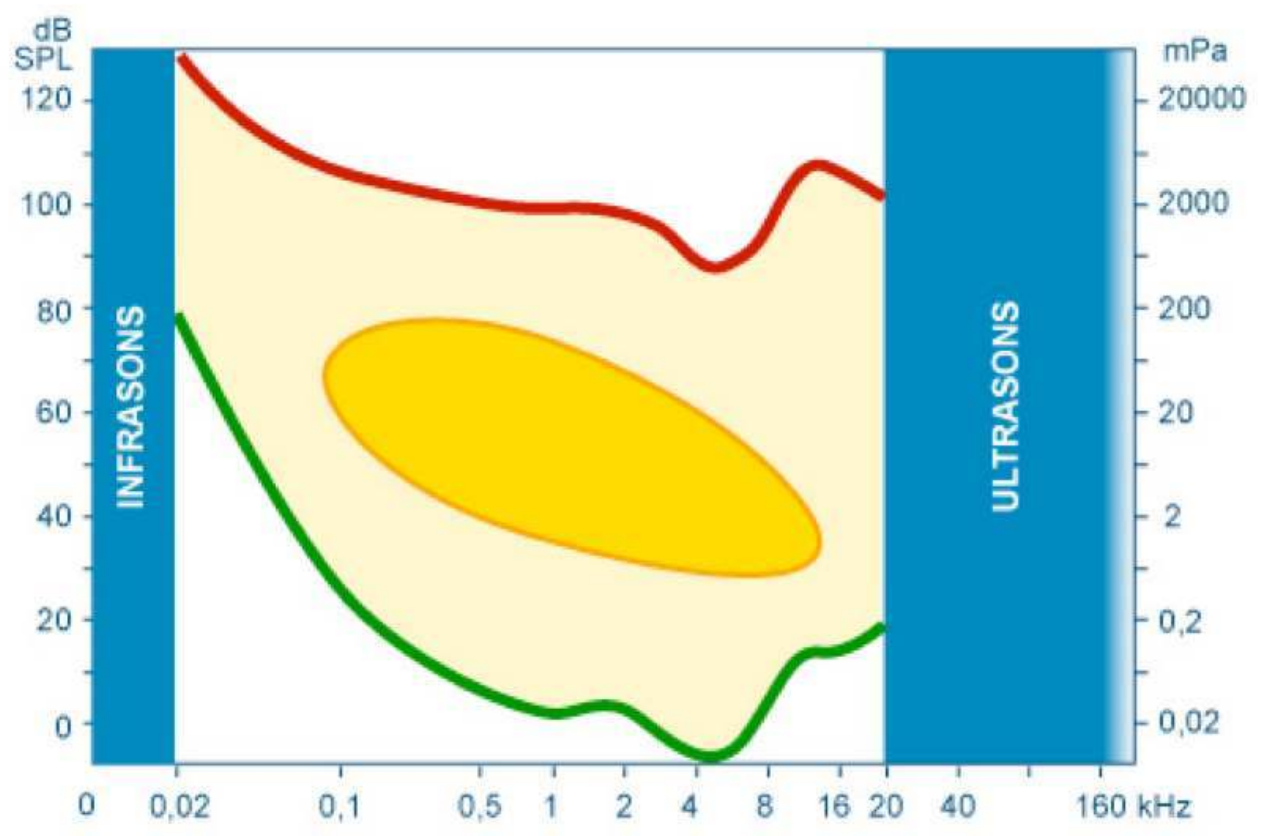

Fig 2 : la perception de la hauteur

http://www.cochlea.eu/son/psychoacoustique

6 Nous sommes en outre inégaux devant l'exercice consistant à identifier précisément la hauteur d'un son, nombre d'auditeurs ne disposant pas de l'oreille absolue. L'être humain est néanmoins, presqu'indépendamment de capacités spécialistes acquises, tout à fait performant dans la perception différentielle de la hauteur. Entre deux sons distincts ou dans la variation d'un même son.

La perception de l'altération du timbre provoquée par l'éloignement, les fréquences élevées se trouvant atténuées par la distance, est donc largement mobilisée dans la représentation des différences de distances entre l'auditeur et les différents objets sonores constituant l'espace dans lequel il est plongé.

7 Si la relation entre grandeur du stimulus et grandeur de la sensation est représentée aisément en terme de niveau et de hauteur, la sensation de localisation est plus difficilement représentée, nous allons tâcher d'éclairer les mécanismes complexes qui sont à l'œuvre. 


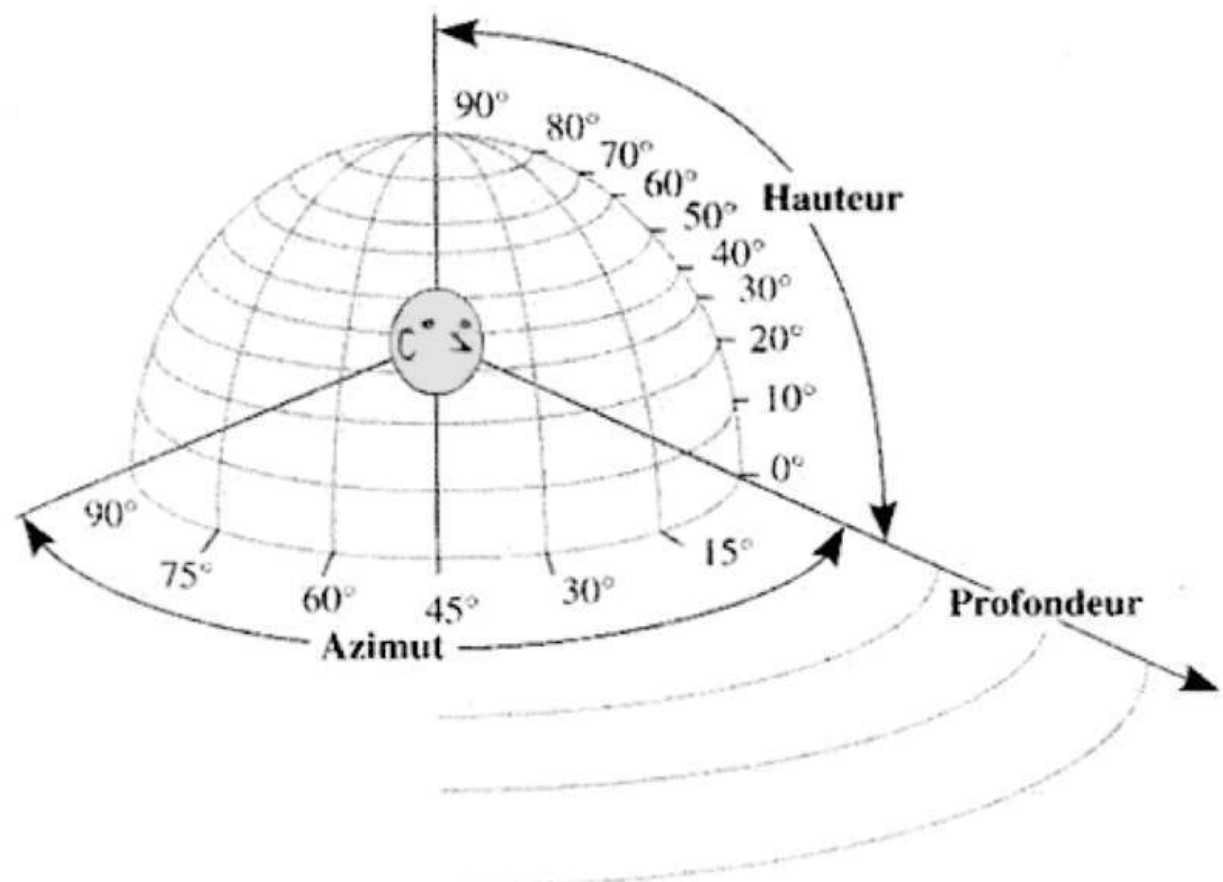

Fig 3 : la perception de la localisation

http://www.studiophebes.com/edu/acoustique/localisation.html

Elle se construit par l'analyse de la différence perçue respectivement par chaque oreille, différence d'intensité et aussi différence de temps.

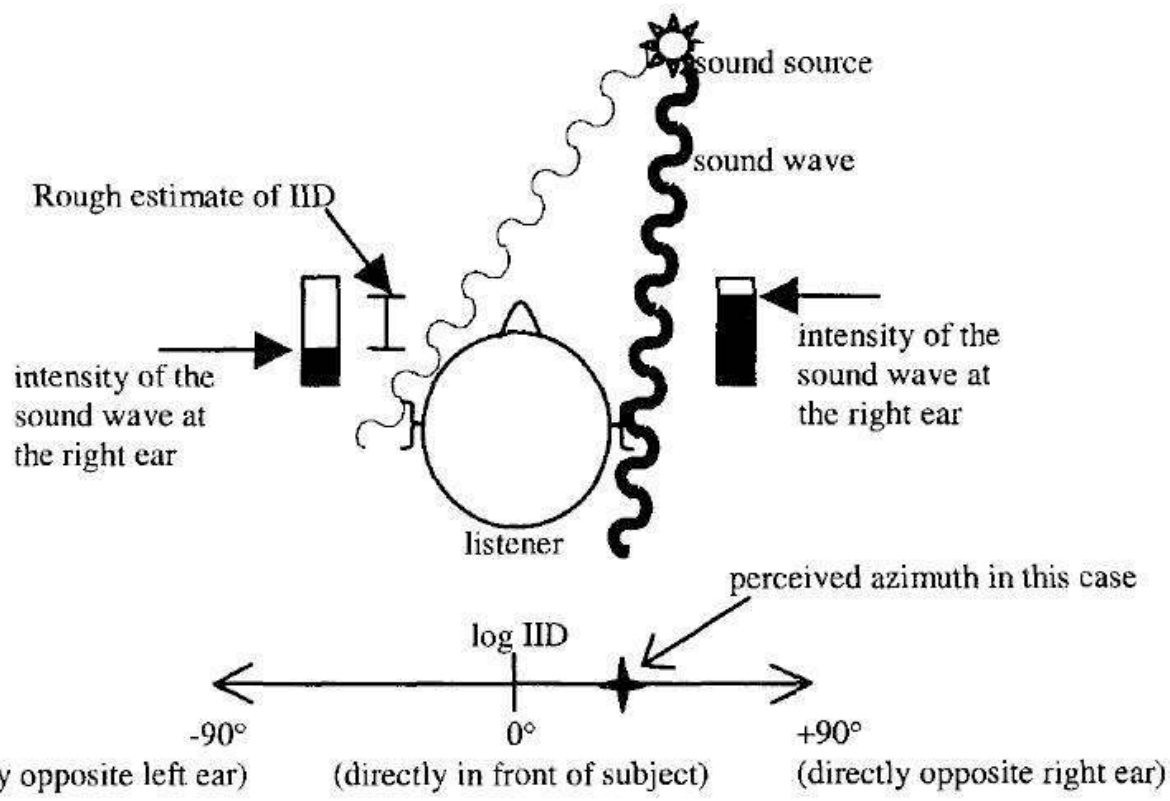

Fig 4 : perception de la différence de temps et d'intensité

http://www.studiophebes.com/edu/acoustique/localisation.html 
Les performances sont bien meilleures dans la localisation horizontale où la sensation de flou couvre une zone de $8^{\circ}$ dans l'axe frontal et $20^{\circ}$ dans l'axe arrière. Sur l'axe vertical, la zone de flou est beaucoup plus importante.

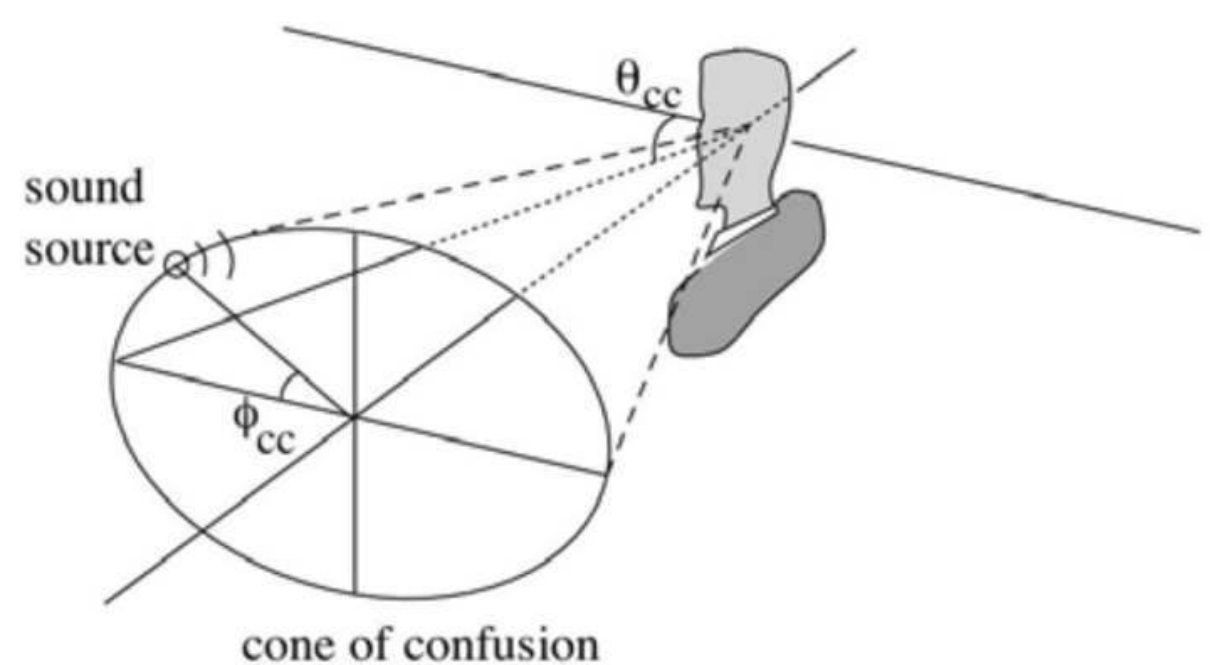

Fig 5 : le cône de confusion de localisation https://rs2007.limsi.fr/PS_Page_16.html

10 Notons ici que la précision de la localisation, dans le cas de l'écoute d'une scène sonore naturelle, voit une contribution très importante apportée par les mouvements de la tête qui vont fournir des informations de triangulation très efficaces à courte distance. Ces informations sont bien sûr inaccessibles lors de l'écoute d'une scène sonore reproduite avec des systèmes de diffusion à base de haut-parleurs. 


\section{L'effet de précédence ou effet Haas}

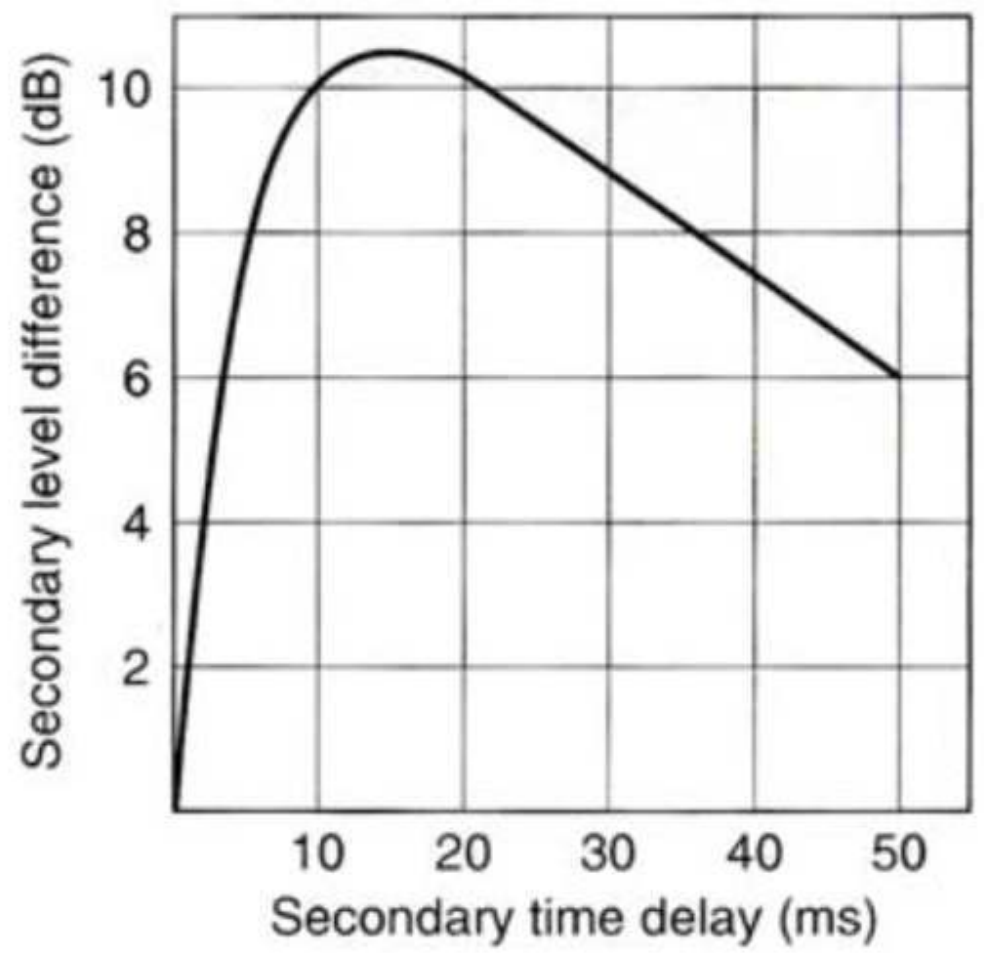

Fig 6 : l'effet de précédence

http://docplayer.fr/174703-Psychoacoustique-vi-localisation-vi-2-lateralisation-differencestemporelles-itds-vi-1-position-du-probleme-vi.html

Lorsque deux sons brefs sont émis dans un intervalle très court, ils sont perçus comme un seul son. L'effet est une augmentation du niveau, indépendamment de la position de la source secondaire. L'intervalle maximum pour que cet effet fonctionne et appelé temps de fusion, il est de l'ordre de $5 \mathrm{~ms}$ pour des transitoires (clic) et de $40 \mathrm{~ms}$ pour les sons plus complexes.

L'effet de précédence joue un rôle très important dans la précision de notre perception naturelle du son, il nous permet de localiser, d'interpréter et d'identifier les sons malgré les transformations qu'ils peuvent subir dans les conditions acoustiques très différentes que nous rencontrons. 
L'effet de masque:

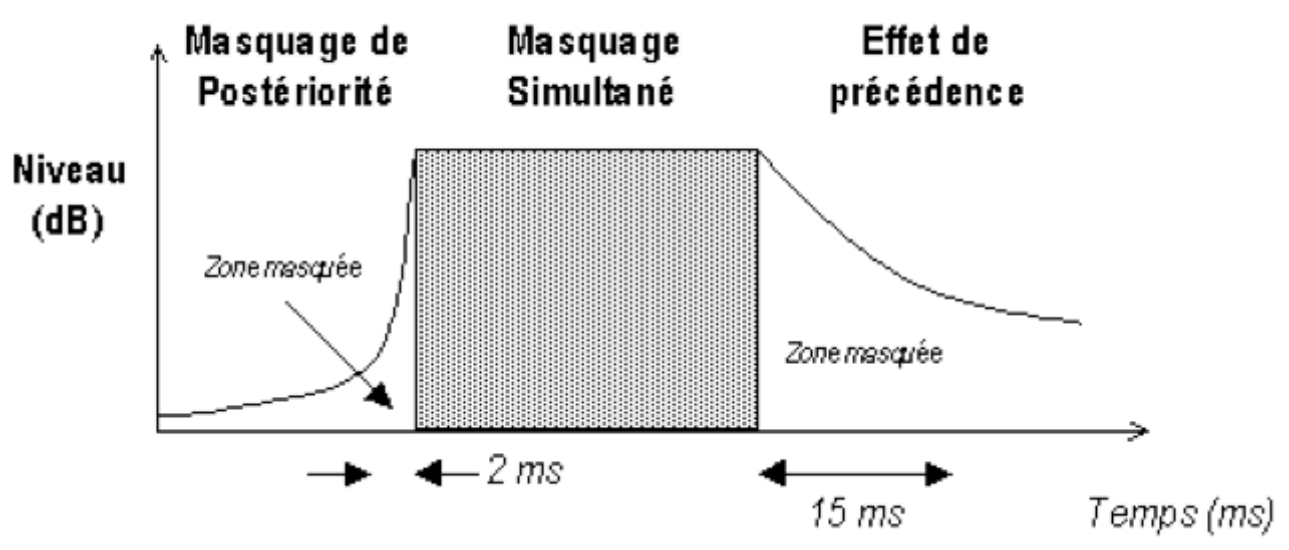

Fig 7 : l'effet de masque

http://www.minidisc.org/French_tech/section1.html

12 Un son masquant de hauteur donné augmente l'intensité que doit avoir un son pour être perçu. Son effet sera maximum pour des sons de hauteur proche ou plus aigus. Un bruit ayant une large répartition de fréquence masquera presque tous les sons. Au delà de son aspect fréquentiel, on observe aussi le phénomène de masquage temporel, le son masquant venant perturber la perception du son masqué, postérieurement mais aussi antérieurement à son apparition.

13 L'observation de ces deux phénomènes perceptifs peut montrer la relation très singulière que la construction de la scène et de l'histoire sonore qui se déroule devant nos oreilles entretient avec la perception et l'analyse des stimuli, dans leur consécution et leur superposition.

L'élaboration d'une sensation de l'espace sonore, de la position et ou des mouvements des objets qui constituent la scène sera d'autant plus accessible que l'identification de ces objets pour s'accomplir sans efforts;

on constate en effet que le processus d'identification de la nature de l'objet sonore est le plus souvent prioritaire, la définition de sa position dans l'espace n'advenant que dans un deuxième temps.

14 Tâchons de définir rapidement les critères de spatialisations caractérisant la position ou la variation de position d'une ou plusieurs sources sonores dans un lieu donné : 


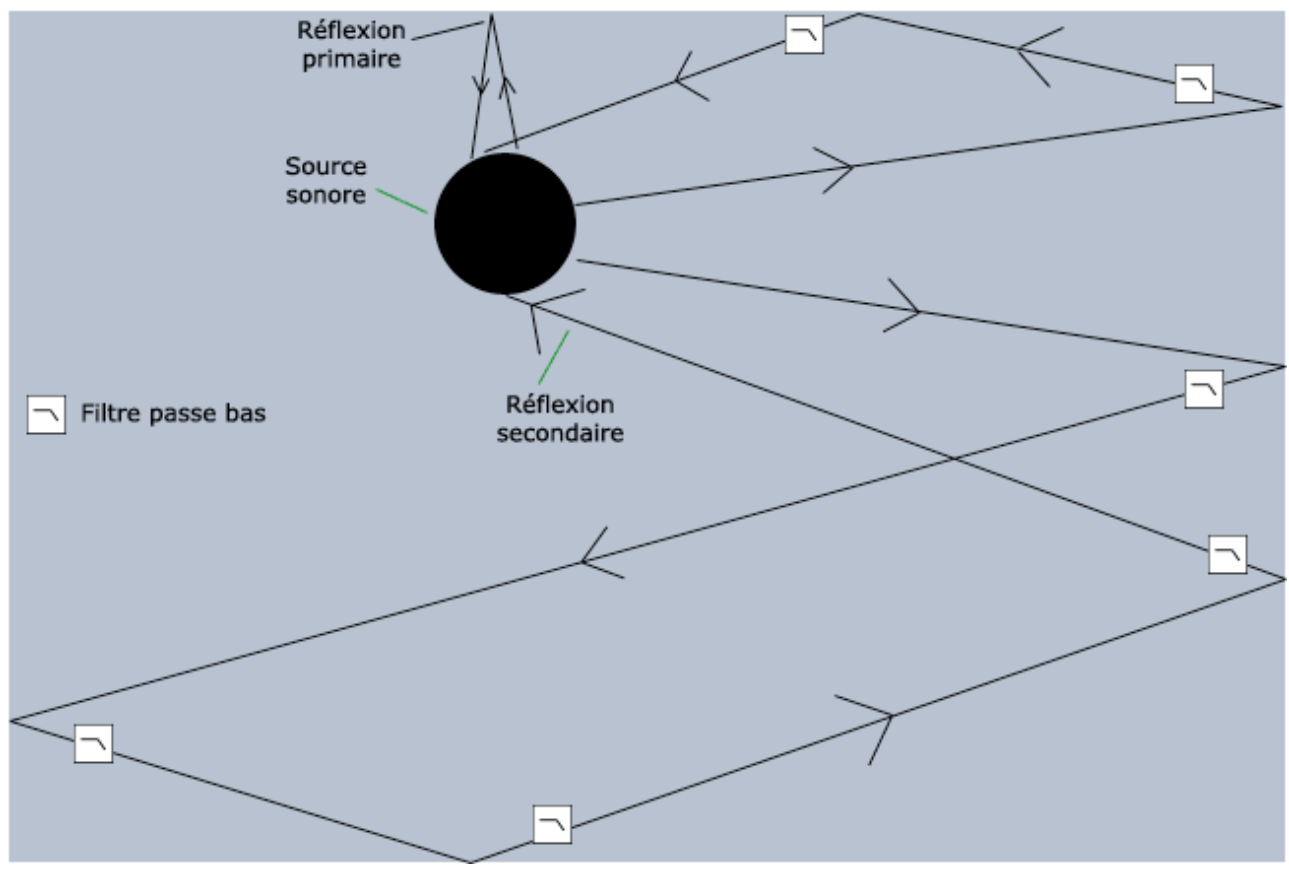

Fig 8 : les différentes réflexions

http://www.majorcom.fr/web/fr/271-les-bases-acoustiques.php

15 - La distance apparente correspond à l'éloignement subjectif (ou intimité) de la source sonore; elle est reliée principalement à 4 paramètres:

* le rapport entre champ direct et champ réverbéré ;

* la distance apparente augmente quand le rapport est au profit du champ réverbéré ;

* les réverbérations précoces (early reflections) sont perçues comme parties intégrantes du son direct et peuvent donc contribuer à la réduction de la distance apparente ;

19 * le niveau sonore, plus il diminue, plus la distance apparente augmente.

* le spectre: si le spectre présente un déficit en hautes fréquences, la distance apparente diminue.

(Ce phénomène est lié à la propagation de l'énergie acoustique dans l'air qui voit les hautes fréquences plus atténuées que les basses fréquences.)

21 Le volume apparent de la salle est principalement lié à la densité des réflexions et au faible retard perçu; plus le retard augmente et plus le contenu spectral est altéré, plus la sensation de volume de la salle augmente.

- l'impression spatiale correspond à la sensation d'être "baigné" dans le son ou à contrario de l'écouter via une "fenêtre" de taille réduite. On peut constater que cette impression est reliée au coefficient d'efficacité latérale, l'énergie réverbérée devant provenir des côtés de la salle ;

23 - l'effet Doppler apporte des informations sur le déplacement d'une source qui s'approche puis s'éloigne de l'auditeur.

Dans notre objectif de représentation de l'espace, nous devrons considérer quelques caractérisant qui provoquent une mobilisation de l'attention auditive:

- la saillance physique : 

de provoquer une réaction de l'orientation de l'attention. Les variations brutales de niveau ou la présence soudaine de fréquences élevées vont le plus souvent attirer l'attention de l'auditeur sur cette source ;

27 - le caractère nouveau, non encore approprié ou identifié par l'auditeur d'un son apparaissant dans la scène sonore va mobiliser une attention particulière.

Par delà les apprentissages spécifiques auxquels procèdent les spécialistes du son, musiciens instrumentistes ou compositeurs, preneurs de son ou mixeurs, on peut constater que, dans un contexte spécifique, la précision de la détection d'une fréquence sonore ou d'une position dans l'espace s'améliore au fur et à mesure que la probabilité d'occurrence augmente. La capacité à discriminer les changements de niveau, de hauteur ou de position dans l'espace dans un pattern complexe masquant augmente à mesure que les incertitudes concernant ce pattern diminuent. On constate donc que à chaque situation d'écoute correspond une opération volontaire d'apprentissage de la scène sonore, cette appropriation fine est destinée à mobiliser les ressources de perception qui nous permettront d'isoler les informations pertinentes. Ce phénomène est largement utilisé dans les opérations de mixage, la possibilité nous étant offerte de diminuer la présence d'un objet sonore dès lors que l'auditeur en a fait l'identification, une baisse significative de son niveau n'affectera pas la sensation de sa présence.

Même si les procédés de diffusion visent plus à provoquer une sensation d'immersion de l'audio-spectateur que de lui donner des informations auditives aptes à décrire précisément les données spatiales de la scène sonore,

les opérateurs de prise de son tentent d'utiliser des dispositifs destinés à capter le plus précisément la totalité de l'espace sonore.

30 Sont utilisés depuis de très nombreuses années des couples stéréophoniques utilisant séparément ou conjointement les différences d'intensité ou de temps. 

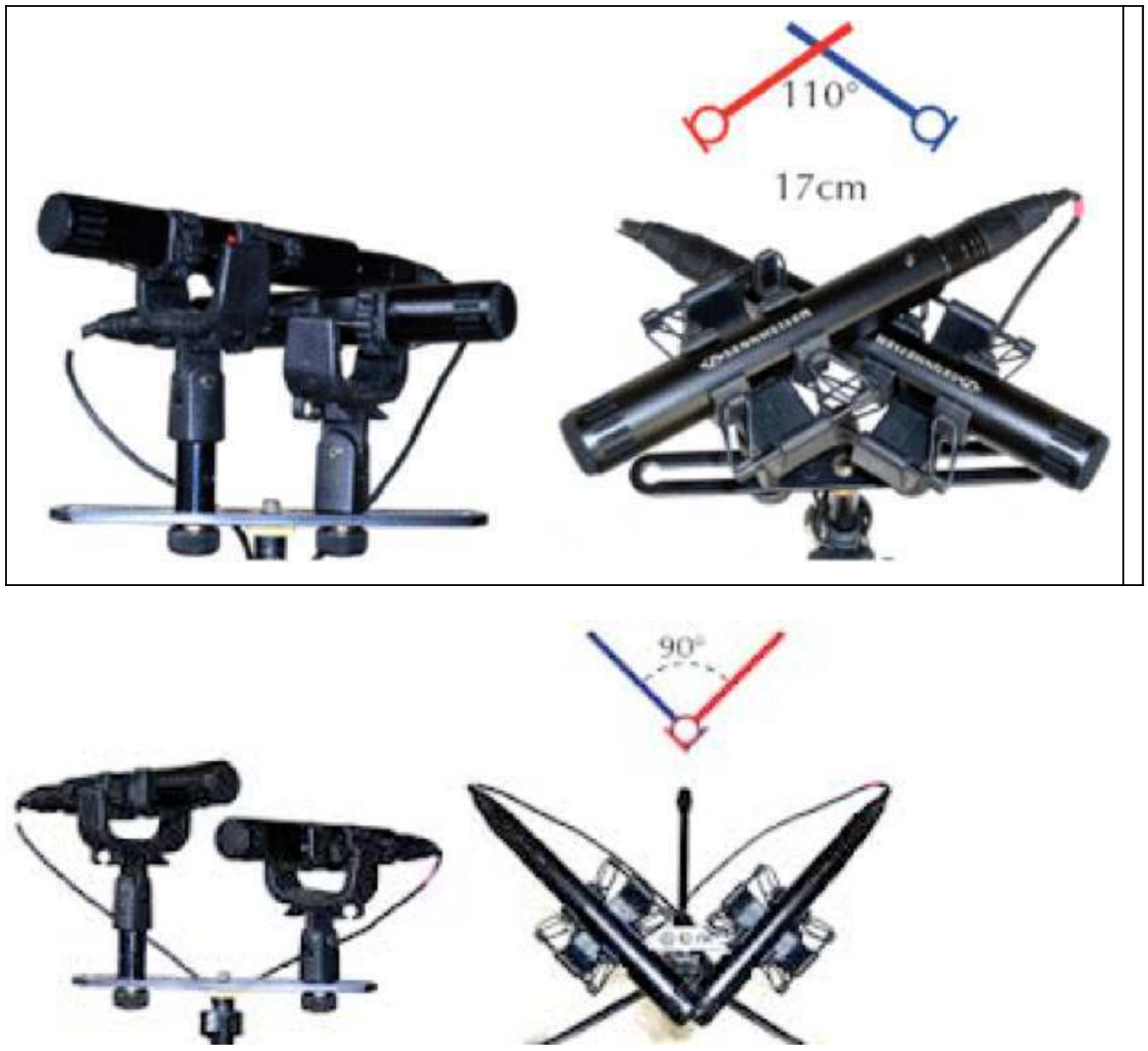

Fig 9 : couple $A B$, couple $X Y$

http://www.dcaudiovisuel.com/product_info.php/products_id/74

Plus récemment sont apparus des dispositifs dotés de 3 microphones LCR destinés à enregistrer 3 canaux discrets. 


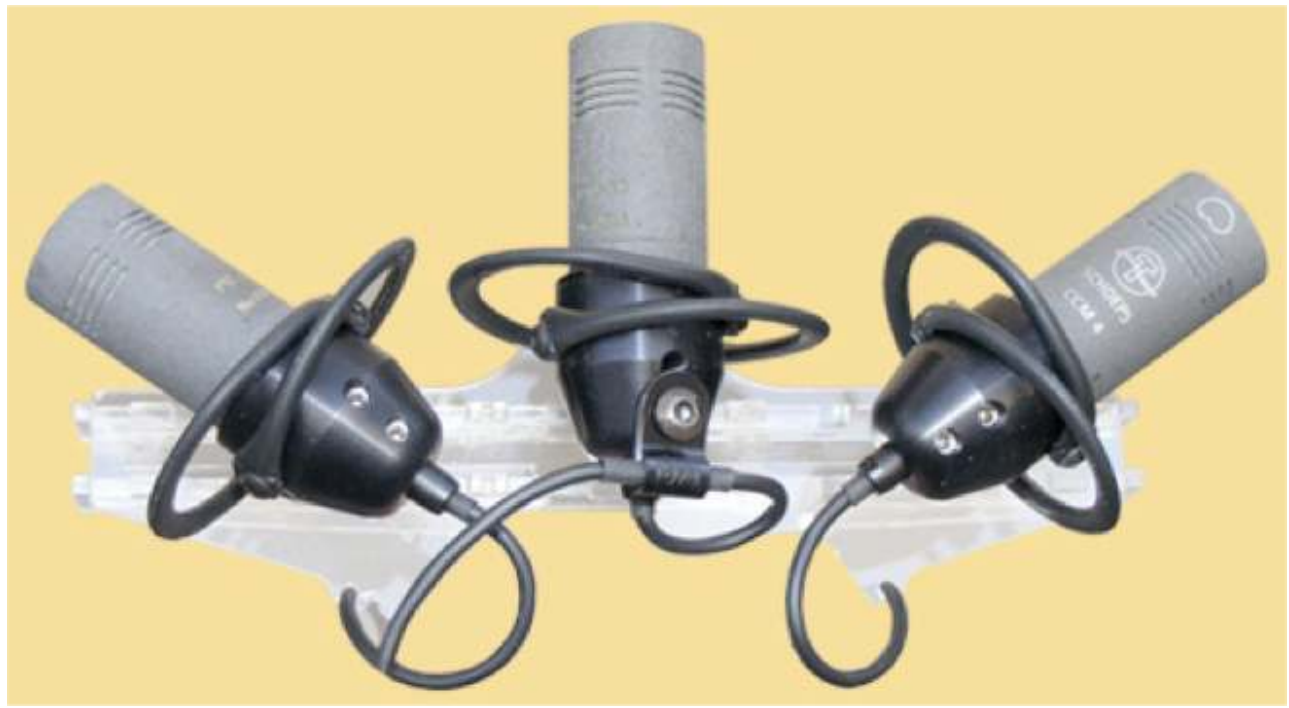

Fig 10 : dispositif de captation LCR

http://www.cinela.fr/albert.php?language =fr

L'utilisation des systèmes de diffusion multicanal a encouragé les preneurs de son à expérimenter l'utilisation de systèmes de captation présentant le même nombre de canaux discrets et l'on a vu apparaitre de nombreuses propositions techniques.

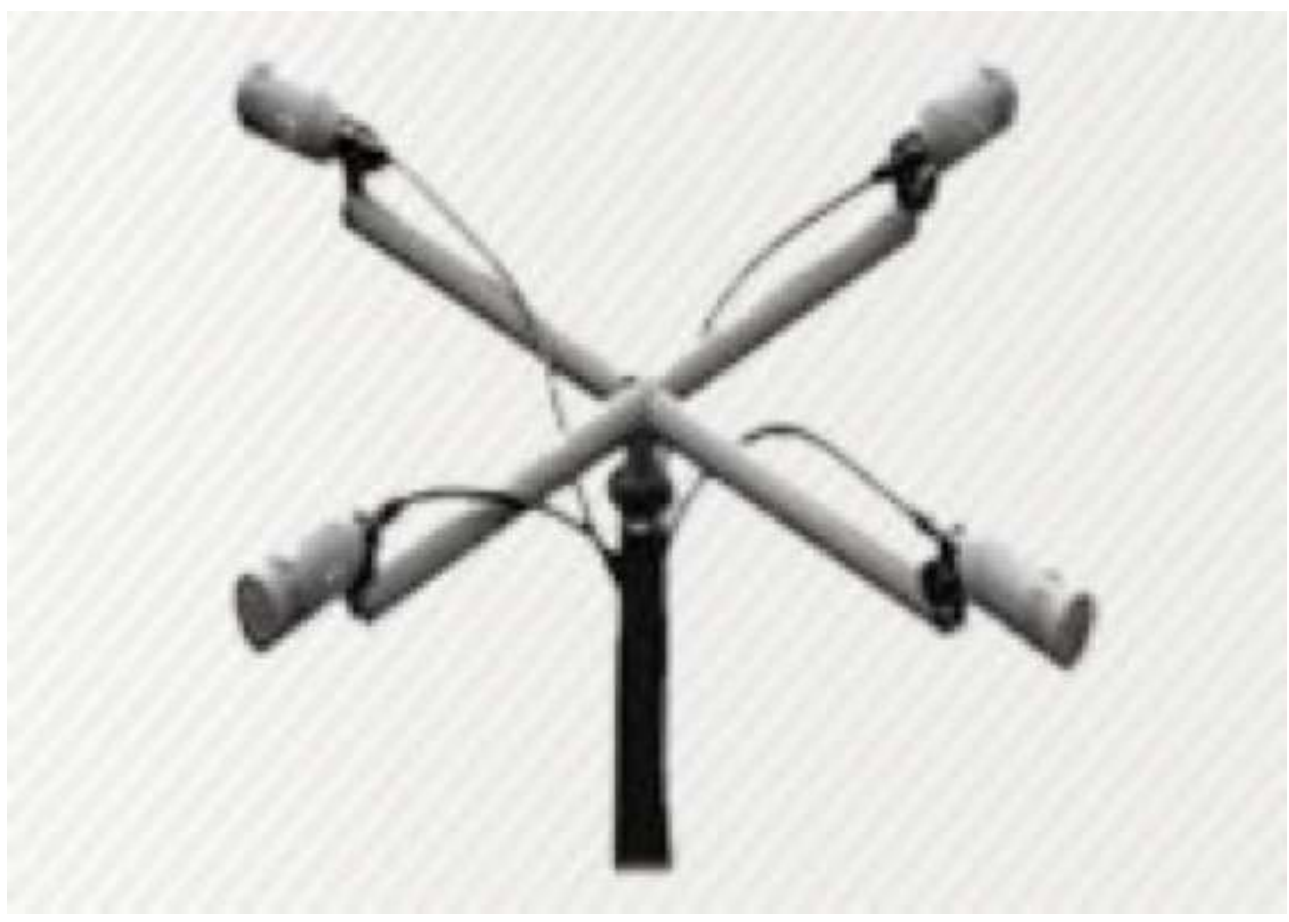

FIg. 11 : CROIX IRT

http://areitec.fr/categorie-produit/catalogue/microphones-catalogue/microphones-surroundmicrophones-catalogue/ 


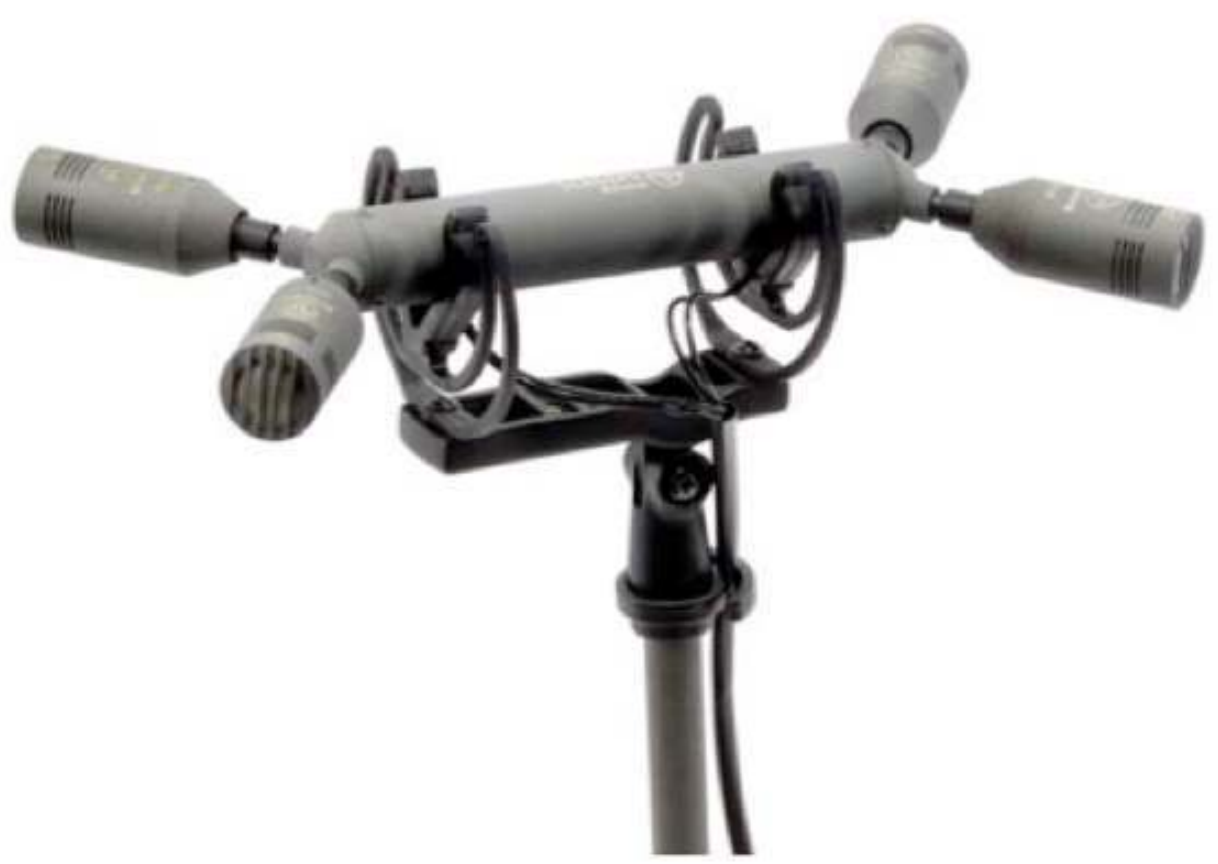

Fig. 12 : Ensemble double AB

http://areitec.fr/categorie-produit/catalogue/microphones-catalogue/microphones-surroundmicrophones-catalogue/ 


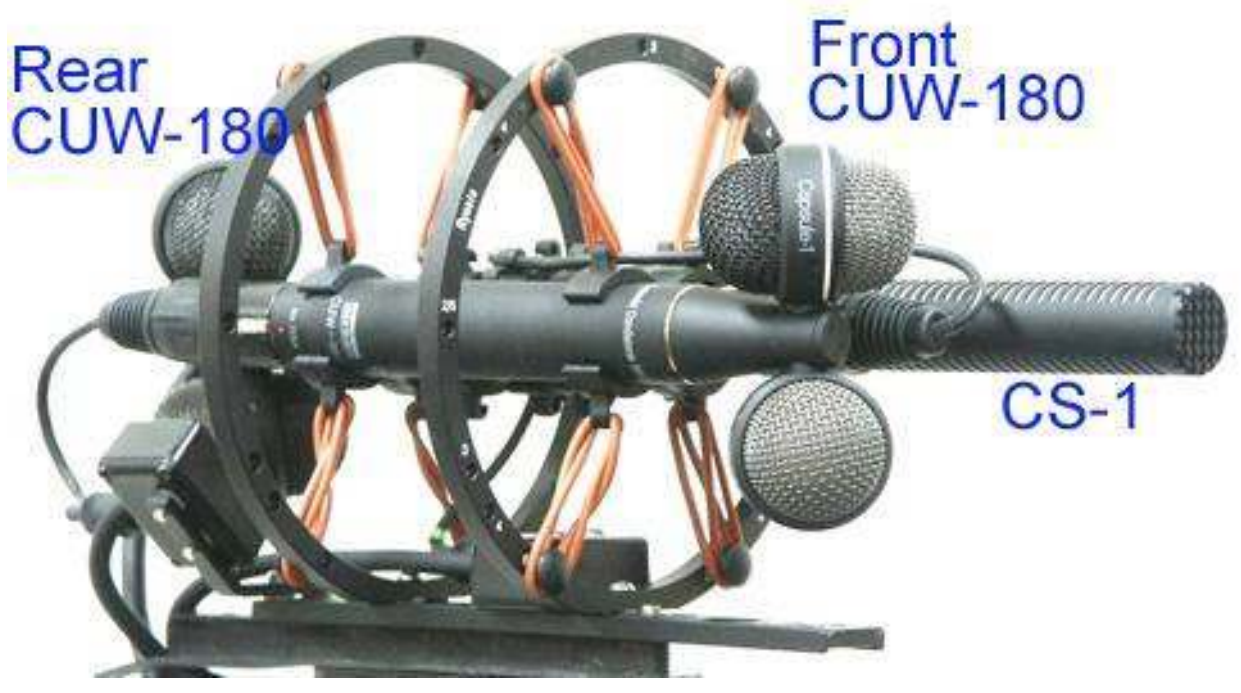

Fig. 13 : Ensemble «surround»

http://areitec.fr/categorie-produit/catalogue/microphones-catalogue/microphones-surroundmicrophones-catalogue/

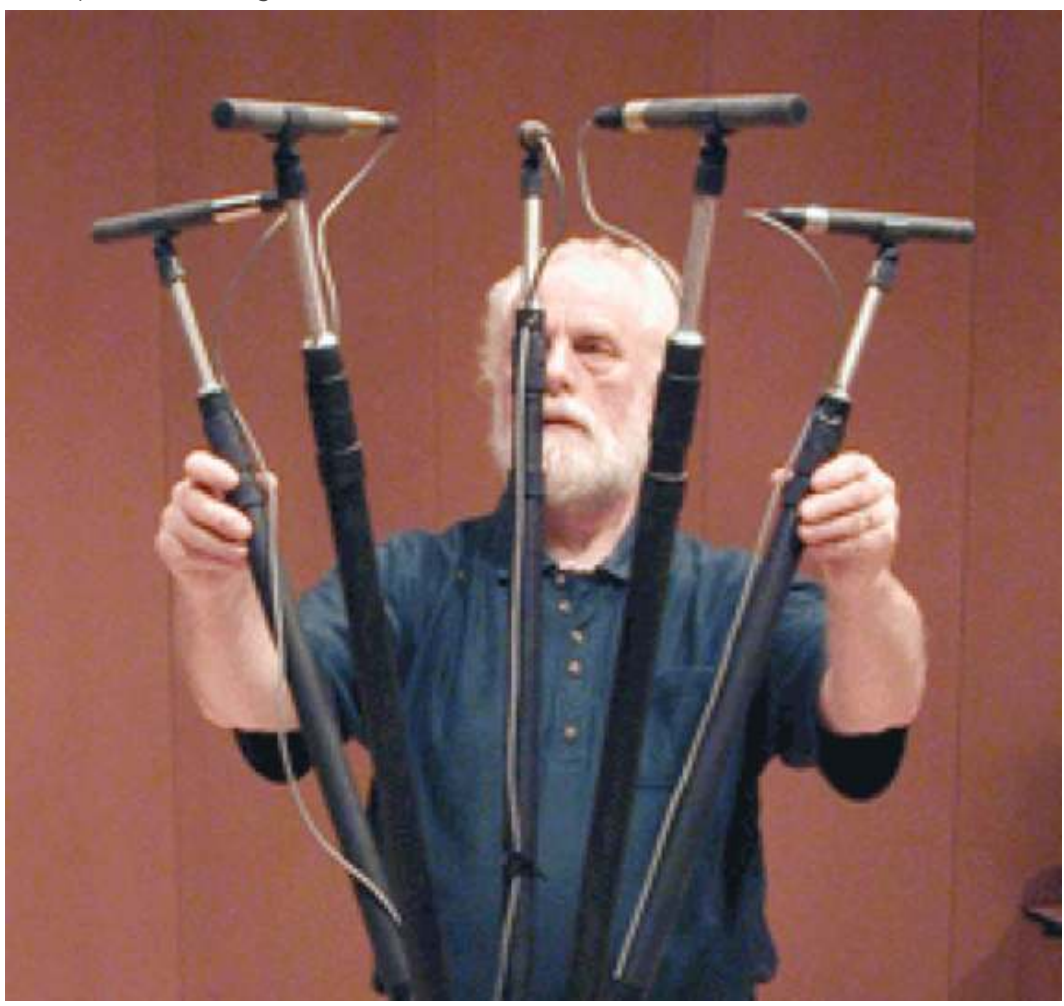

Fig. 14 : Mike Williams

http://www.retifweb.net/?var=10047\&part=2 


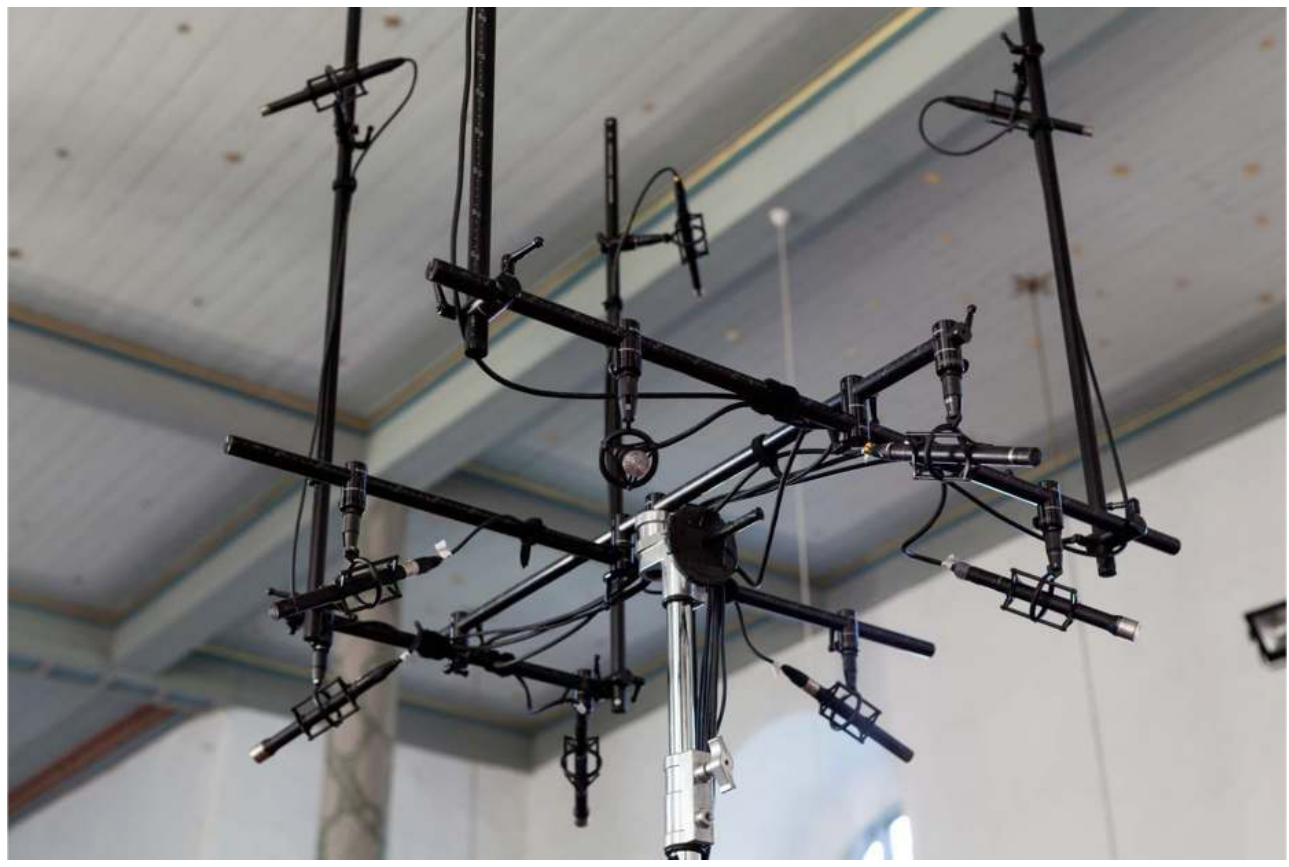

Fig. 15 : Complexe...

http://www.lesonmulticanal.com/category/prise-de-son/

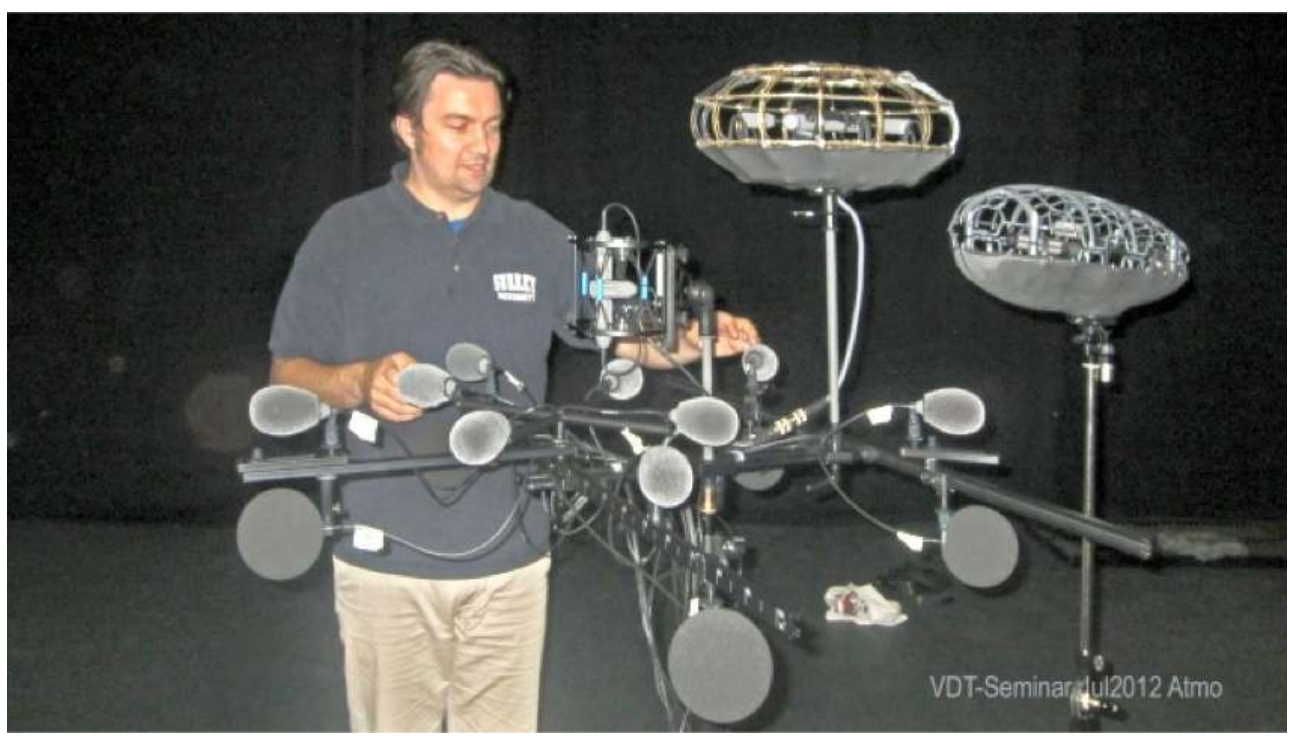

Fig. 16 : mais encombrant !

http://www.lesonmulticanal.com/category/prise-de-son/

Ces démarches d'abord expérimentales puis opérationnelles s'inscrivent dans une perspective holophonique de la prise de son. Elles s'avèrent souvent incompatibles avec les contraintes du tournage et en outre d'un apport assez limité en terme de satisfaction de l'auditeur tant les dispositifs de diffusion au cinéma ou en home cinéma sont encore inadaptés.

Pour autant, les éléments fournis aux monteurs sons et mixeurs grâce à ces procédés sont tout à fait précieux et, même si leur utilisation nécessite un soin particulier, ils permettent un travail de représentation de la scène sonore tout à fait enrichi. 
Évoquons rapidement les systèmes de diffusion sonore généralement rencontrés dans les diverses situations d'écoute.

On peut constater que si le cinéma en 3D nécessitant l'utilisation de lunettes à polarisant s'est largement répandu, nous sommes restés en matière de diffusion sonore dans une logique d'enveloppement de l'auditeur plus que d'offre d'une sensation plus précise de l'espace représenté.

3 L'impératif de l'homogénéité de couverture sonore de l'auditoire dans une salle de cinéma ou de concert est à bien des égards parfaitement incompatible avec une précision de localisation des sources sonores indispensable à la réception satisfaisante de l'espace sonore.

37 Comme nous avons pu le constater, l'appareil auditif, effectue une analyse extrêmement fine des différences temporelles et spectrales des stimuli lui parvenant.

Cette finesse est le résultat d'un apprentissage complexe et mobilise la perception en intégrant les singularités de l'appareil auditif mais aussi du lieu d'écoute.

8 Un dispositif de diffusion très efficace en terme de précision de localisation et de sensation de l'environnement est sans doute la restitution binaurale intégrant une fonction de transfert relative à la tête;

La technologie binaurale est basée sur la diffusion d'un couple de signaux binauraux, l'un étant destiné au canal droit, l'autre au canal gauche. Les signaux binauraux peuvent être obtenus de deux manières : par la prise de son directe, ou par calcul du signal, en filtrant un signal monophonique au travers des filtres binauraux qui reproduisent les propriétés de la propagation acoustique entre une source placée dans une position donnée et les deux oreilles de l'auditeur.

39 Ces filtres, résultant de la fonction de transfert de la tête de l'auditeur doivent être conçus spécifiquement pour chaque individu. On comprendra aisément les difficultés de mise sur le marché d'une telle technologie même si les tentatives de modélisation réduite à quelques modèles standard sont pleines de promesses, un résultat satisfaisant ne peut que nécessiter la prise d'une empreinte individuelle.

Ce dispositif peut fonctionner en utilisant un casque stéréophonique de bonne qualité mais présente les meilleures performances en utilisant des écouteurs moulés précisément selon la morphologie de chaque oreille.

Les dispositifs de diffusion implantés dans les salles de cinéma et qui peuvent également constituer une installation domestique de qualité répondent à des normes instaurées par Dolby, on parle de diffusion "surround". 


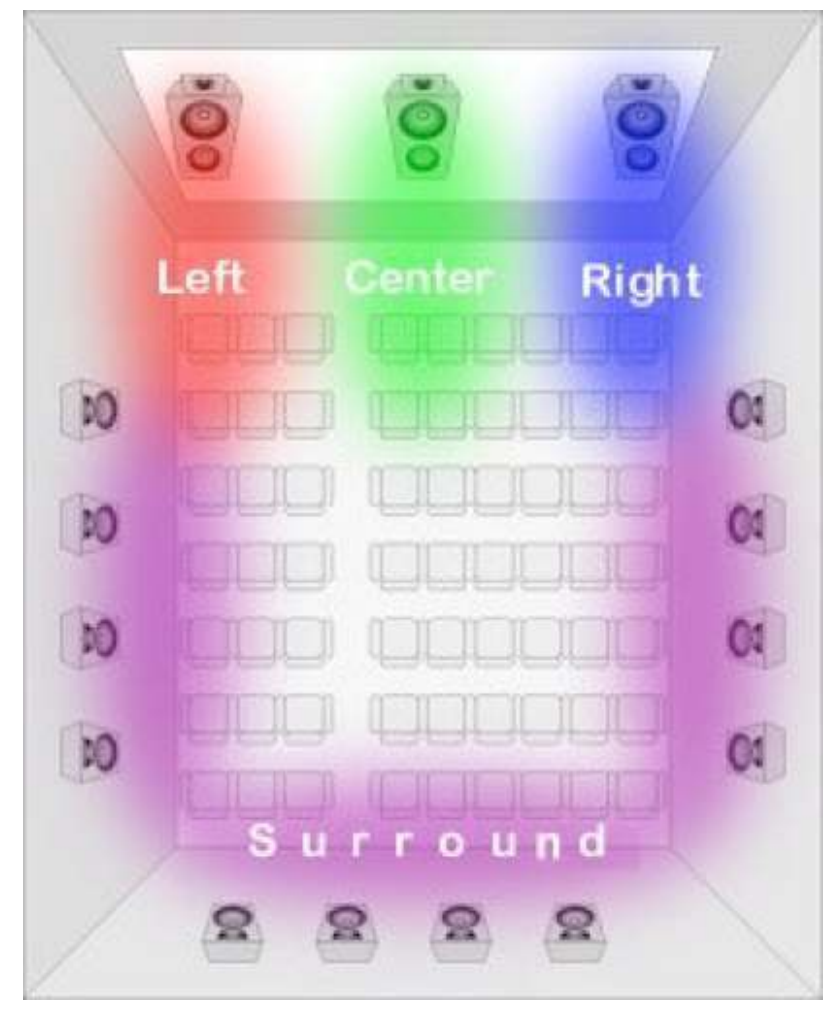

Fig 17 : dolby surround

http://hometheaterhifi.com/volume_8_1/dolby-prologic2-3-2001.html

41 Nous sommes bien en présence d'un système destiné à provoquer une sensation d'enveloppement plus que de préciser la localisation des sources sonores. Notons à ce titre que nombre de réalisateurs restent méfiants devant ce procédé, arguant de la perturbation potentiellement ressentie par l'audio-spectateur percevant une information sonore hors de l'axe de l'image.

On aurait pu penser que le développement du cinéma "3D" aurait ouvert les vannes de nouvelles créativités à cet égard, on constate que ce n'est pour l'instant que très rare.

Lors du passage à la diffusion HD par les télévisions française, il a été demandé aux producteurs des programmes de réaliser le son en multicanal, beaucoup d'entre eux se sont révélés sans inspiration et ont laissé le soin aux mixeurs de faire les choix des sources diffusées sur les différents canaux, sans accorder plus de temps à la conception et à la réalisation de cette bande sonore.

Dolby a présenté récemment un nouveau dispositif de diffusion multicanal ATMOS qui présente un intérêt qui va au delà du nombre de haut-parleurs supplémentaires installés. 


\section{Dolby Atmos}

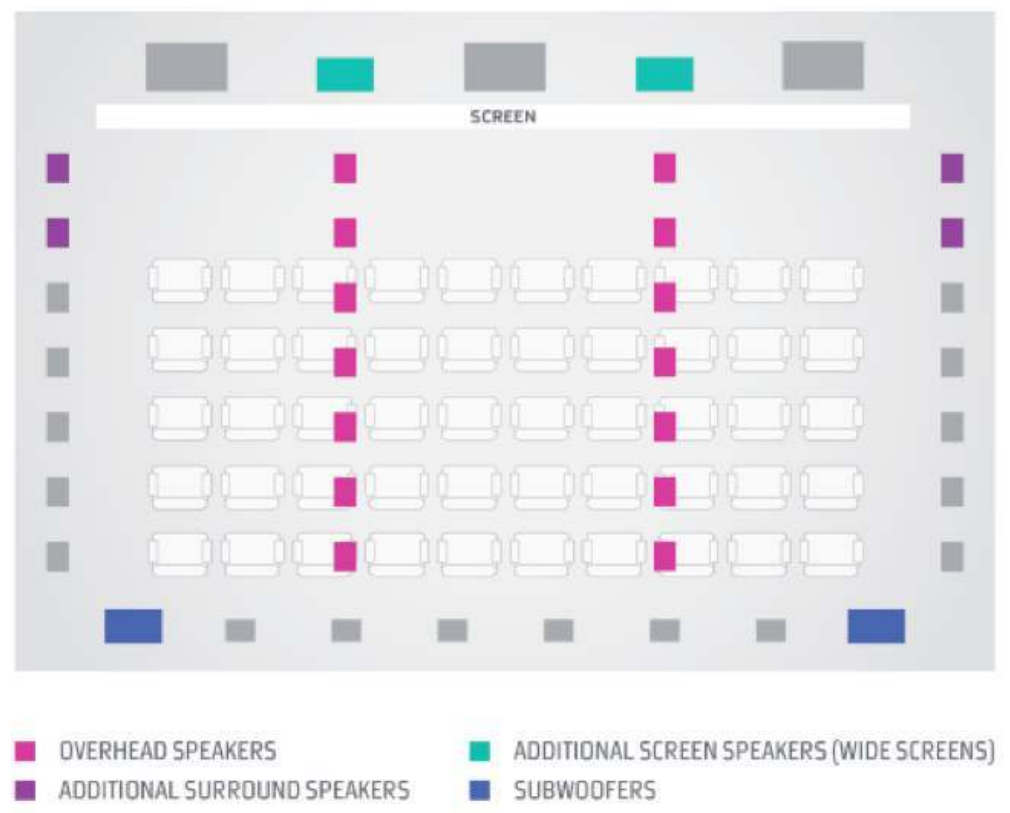

Fig 18 : Dolby Atmos

http://www.extremetech.com/extreme/178670-dolby-atmos-true-3d-surround-sound-around-aboveand-below-finally-comes-of-age

La chaîne de production sonore imposée par ce format ouvre de réelles perspectives en terme de créativité pour le réalisateur et surtout de précision de localisation des sources pour l'audio-spectateur. Un très petit nombre de salles sont équipés et il est trop tôt pour juger du développement de ce procédé dans les productions cinématographiques, seules celles disposant de budgets confortables s'y risqueront quoi qu'il en soit.

Un autre procédé de diffusion est tout à fait séduisant en ce qu'il propose une restitution de la position des sources sonores conforme à la réalité:

le système WFS.

Il s'agit d'une implémentation audio du principe de Huygens-Fresnel, qui spécifie que: "Tout champ sonore émergeant d'une source principale peut être reproduit en sommant les contributions d'une distribution infinie et continue de sources sonores secondaires". D'un point de vue théorique, la WFS permet donc de synthétiser des sources sonores à n'importe quelle position. En pratique, nous sommes limités au plan horizontal. 


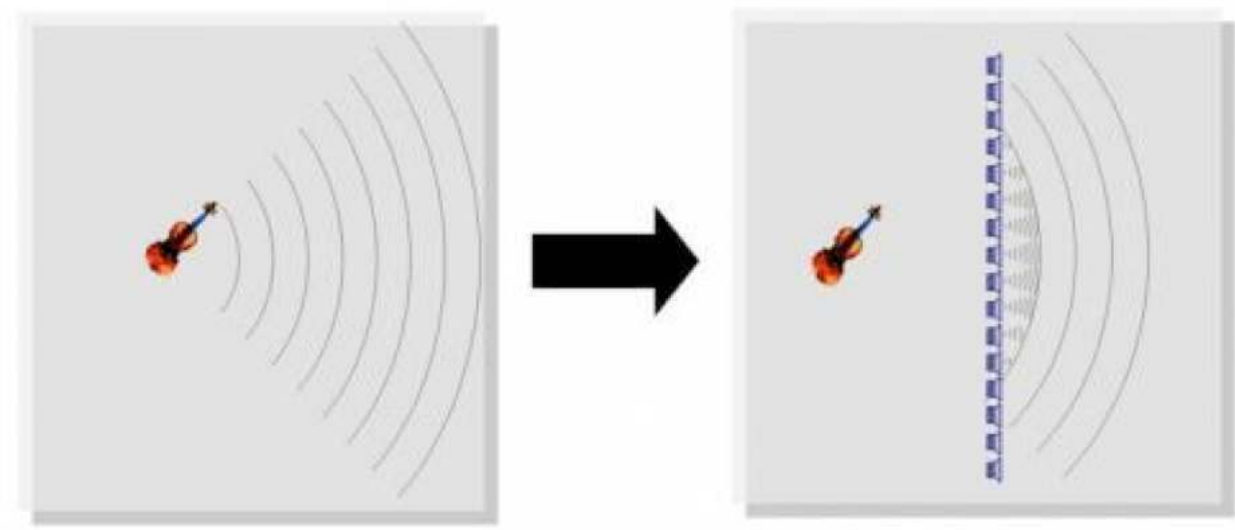

Fig 19 : principe de la WFS

https://acousticengineering.wordpress.com/2013/12/08/wavefield-synthesis-souped-up-surroundsound/

La technique Wave Field Synthesis (WFS) désigne un procédé de reproduction holophonique qui permet, par analogie avec les hologrammes visuels, de capter ou synthétiser une scène sonore en préservant les informations spatiales de distance et de direction des sources qui la composent. Cette approche, initiée par l'université de Delft, dépasse les limites des systèmes conventionnels en termes de fidélité de reproduction sur une zone d'écoute étendue.

Tandis que les techniques stéréophoniques conventionnelles (stéréo, 5.1) s'apparentent au trompe-l'œil et ne peuvent ainsi être appréciées que depuis le centre du dispositif, l'holophonie a l'ambition de reconstruire un champ sonore dans lequel les auditeurs peuvent se déplacer en gardant une perception cohérente de la localisation des sources.

Les ressources techniques de représentation synthétiques de l'espace sont bien sûr très présentes dans les studios de mixage et nous ne pouvons les détailler ici au risque de déborder de l'espace qui nous est offert.

On ne compte plus les dispositifs de spatialisation artificielle, et depuis très longtemps, la réverbération artificielle, fait partie des outils les plus utilisés.

Les processeurs de spatialisation peuvent désormais intégrer l'empreinte acoustique d'un lieu pour l'appliquer ensuite à une source sonore.

On constate cependant que cette transposition de la figuration de l'espace est moins efficace qu'on pourrait l'attendre, il faut souvent aménager le réglage des différents paramètres pour obtenir un résultat satisfaisant.

La difficulté des opérations de mise en espace réside également dans la nécessité de considérer les conditions d'écoute de l'auditeur final.

D'un point de vue acoustique, celles-ci sont normalisées dans le cas des salles de cinéma mais c'est loin d'être le cas des installations domestiques et plus encore des situations d'écoute nomade.

52 Cette rapide présentation avait pour objectif d'éclairer les enjeux de la relation que les "faiseurs de son", de la prise de son jusqu'à la diffusion, entretiennent avec la notion d'espace et de perspective sonore.

Comme dans toute activité artisanale, il s'agit pour nous d'acquérir les connaissances les plus fines des modalités de construction de la représentation d'un espace sonore par l'auditeur en s'appuyant sur des données scientifiques et techniques les plus larges possibles sans jamais rien céder à la joyeuse liberté de la recherche et de 
l'expérimentation qui, nous en sommes convaincus, est la meilleure garantie du plaisir de la réception chez l'audio-spectateur auquel sont destinés nos travaux.

\section{BIBLIOGRAPHIE}

Marie-Claire Botte, Psychoacoustique et perception auditive, Tec \& Doc, 1999 (ISBN 978-2852065345)

Georges Canevet, « Audition binaurale et localisation auditive : aspects physiques et psychoacoustiques », dans Botte \& alii, Psychoacoustique et perception auditive, Paris, Tec \& Doc, 1999

Mpaya Kitantou, « La perception auditive », dans Denis Mercier (direction), Le Livre des Techniques du Son, tome 1- Notions fondamentales, Paris, Eyrolles, 1987, 1e éd

Stephen McAdams (dir.) et Emmanuel Bigand (dir), Penser les sons : Psychologie cognitive de l'audition, Paris, PUF, coll. « Psychologie et sciences de la pensée », 1994, 1e éd., 402 p. (ISBN 2-13-046086-0)

Pierre Schaeffer, Traité des objets musicaux : Essai interdisciplines, Paris, Seuil, 1977, 2e éd. (1re éd. 1966), 713 p.

B. Gardner, "A real-time multichannel room simulator", 124th meeting of the Acoustical Society of America (New Orleans), 1992.

J.-M. Jot, "Etude et realisation d'un spatialisateur de sons par modeles physiques et perceptifs", These de doctorat, Telecom Paris, 1992.

Sivonen, V. P., and Ellermeier, W. (2006). "Directional loudness in an anechoic sound field, headrelated transfer functions, and binaural summation", J. Acoust. Soc. Am. 119, 2965-2980.

V. R. Algazi and R. O. Duda,"Headphone-based spatial sound," IEEE Signal Processing Magazine, Vol. 28, No. 1, pp. 33-42,Jan. 2011.

V. R. Algazi and R. O. Duda, "Effective Use of Psychoacoustics in Motion Tracked Binaural audio ," ISM 2008 (Proc. Tenth IEEE International Symposium on Multimedia), pp. 739-746,pp. 562-567, Berkeley, CA (Dec. 2008).

R. O. Duda, "Modeling head related transfer functions," in Proc. Twenty-Seventh Annual Asilomar Conference on Signals, Systems and Computers (Asilomar, CA, Nov. 1993).

Webographie

Groupe audio acoustique du CNRS

https://groupeaa.limsi.fr/thmsonesp:start

Franck Ernould

http://www.ernould.com/

IRCAM

http://www.ircam.fr/

Michel Vanoy "acousmates"

http://www.studiophebes.com/edu/Accueil.html 
Conservatoire National des Arts et Métiers Département d'acoustique

http://www.duanrevig.com/Acoustique.htm

CIPIC/IDAV Interface Laboratory University of California

http://interface.cipic.ucdavis.edu/sound/

Cosmic Garden

http://cosmicgarden.eu/portfolio/experience-binaurale

Euphonia

http://euphonia.fr/index.htm

Dolby

http://www.dolby.com/fr/fr/index.html

http://www.lesonmulticanal.com/category/prise-de-son/

http://binaural.jimdo.com/limites-du-virtuel/l-oreille-interface-de-r\%C3\%A9alit\%C3\%A9-

virtuelle/synth\%C3\%A8se-binaurale/

\section{RÉSUMÉS}

Il s'agit ici de proposer un aperçu de quelques techniques mises en œuvre dans l'objectif de proposer à l'auditeur, ou l'audio-spectateur une représentation convaincante de la notion d'espace sonore.

Après une description des sensations éprouvées qui concourent à une représentation de l'espace, nous évoquerons quelques procédés techniques et leurs perspectives.

After a concise statement of the auditory perception, an overview of some audio production processes implemented in order to cogently figure sound space in filmmaking.

\section{INDEX}

Mots-clés : Cinéma, espace, écoute, multicanal, prise de son

Keywords : Cinéma, espace, écoute, multicanal, prise de son

Palabras claves : Cinéma, espace, écoute, multicanal, prise de son

Schlüsselwörter : Cinéma, espace, écoute, multicanal, prise de son

\section{AUTEUR}

\section{A. BEZNOSIUK}

Ingénieur du son et enseignant à l'École Supérieure d'AudioVisuel (ESAV) à Toulouse.Chercheur associé au LARA SEPPIA.Les thèmes de son travail de recherche et d'expérimentation sont :L'écoute spécialiste des professionnels du sonLes interfaces utilisateurs des systèmes de mixage et de traitement audio 